\title{
Comparative Study between Laparoscopic Sleeve Gastrectomy and Laparoscopic Mini Gastric Bypass in Control of Type 2 Diabetes Mellitus in Obese Patients Ashraf Abd-Elhamid Abd-Elmonem ${ }^{1}$, Kamel Suliman Hamad ${ }^{2}$, Mohamed Noshi El-Alfi ${ }^{3}$, Abd El-Hafez Abd-El Aziz Selim ${ }^{1}$, Fouad Ebrahim Fouad Agwa* \\ ${ }^{1}$ General Surgery Department, ${ }^{2}$ Clinical Pathology Department, ${ }^{3}$ Internal Medicine Department \\ Faculty of Medicine, Al-Azhar University \\ *Corresponding author: Fouad Agwa , E-Mail: foadagwa@gmail.com
}

\begin{abstract}
Background: Among morbidly obese adult patients (body mass index (BMI) $>40 \mathrm{~kg} / \mathrm{m} 2$ ) those who are super obese $\left(B M I>60 \mathrm{~kg} / \mathrm{m}^{2}\right)$ present particular challenges for bariatric surgeons. Management of super obese (SO) patient has been associated with higher morbidity and mortality and increase surgical risk. The optimal surgical management of these patients is controversial. Aim: This work aimed to focus light on super obese patient. Considering the advantages, the disadvantages and determine long-term outcome of different plans of management regarding the recent guidelines for this group of patients. Patients and Methods: This study included 60 obese patients with type 2 diabetes mellitus (DM) and randomly divided using closed envelopment method into two groups: Group (1): (30 patients) treated by laparoscopic Sleeve gastrectomy (LSG), Group (2): (30 patients) treated by laparoscopic Mini-Gastric Bypass (MGB). Results: This study included 60 obese patients with type 2 D.M. As regard all sample patients (60 obese patients with type 2 D.M), the group age ranged between $22-55$ years with a mean \pm SD of $37.88 \pm 9.52$ years. The group BMI ranged between $37-72 \mathrm{~kg} / \mathrm{m}^{2}$ with a mean \pm SD of $52.42 \pm 9.45 \mathrm{~kg} / \mathrm{m}^{2}$. Of the 60 patients, 38 patients $(63.3 \%)$ were women and 22 patients (36.7\%) were men. Conclusion: Bariatric surgery (LSG and MGB) is not only weight reducing surgery but a metabolic surgery which can cure most of the metabolic syndrome and they are considered the most effective long term treatment modality for type 2 diabetes in obese patients and with comparing between LSG and MGB, our study suggests that MGB has better and earlier effect than LSG in diabetes remission.
\end{abstract}

Keywords: Laparoscopic Sleeve Gastrectomy, Laparoscopic Mini Gastric Bypass, Type2 Diabetes Mellitus, Obese Patients.

\section{INTRODUCTION}

Among morbidly obese adult patients $(\mathrm{BMI}>40 \mathrm{~kg} / \mathrm{m} 2)$, those who are super obese $\left(\mathrm{BMI}>60 \mathrm{~kg} / \mathrm{m}^{2}\right)$ present particular challenges for bariatric surgeons. Management of super obese (SO) patient has been associated with higher morbidity and mortality and increased surgical risk. The optimal surgical management of these patients is controversial. Obesity is a multifactorial condition that arises as a result of genetic, cultural, social, and dietary factors ${ }^{(\mathbf{1})}$.

In the USA, the obesity epidemic has reached record numbers, with greater than $30 \%$ of the adult population being obese, and twice that number experiencing overweight or obesity ${ }^{(2)}$.

With these alarming percentages, the management of obesity has become a public health priority and many options for weight loss are available for this population. Surgical intervention has been shown to be the most reliable and popular way to treat morbidly obese patients struggling with conservative treatments such as diet and exercise $^{(2)}$.

Among the population of morbidly obese adult patients (BMI $>40 \mathrm{~kg} / \mathrm{m} 2$ ), super obese (BMI $>60 \mathrm{~kg} / \mathrm{m} 2$ ) patients present particular challenges for bariatric surgeons. Among the common technical difficulties related to the size of super obese patients is that surgical navigation is more complex ${ }^{(1)}$.

Moreover, thicker layers of abdominal wall and intraabdominal fat, longer distance between the xiphoid and the esophagus), and massive hepatomegaly are some of the surgical hindrances associated with this patient population. Surgical treatment of super-super obese patient has also been associated with higher surgical morbidity and mortality and increased surgical risk (3). In addition, higher BMI at the time of surgery has been linked with higher incidence of major surgical complications for certain bariatric procedures, as well as longer length of hospitalization, increasing rates of 30-day readmission, and rising treatment costs ${ }^{(\mathbf{1})}$.

Initial surgical management options for these patients include the well-established and widely accepted Roux-en-Y gastric bypass (RYGB) surgery as well as sleeve gastrectomy (LSG), a procedure that has gained recent popularity due to its simplicity and favorable complication profile ${ }^{(\mathbf{1})}$.

When deciding which type of bariatric surgical procedure the most appropriate is for super obese patients, cost, operative time, presurgical comorbidities, and experience of the surgeon have to be considered. The rate of post- 
operative complications and the incidence of metabolic complications make the duodenal switch procedure less attractive, and it is performed in a small percentage of surgical patients ${ }^{(2)}$.

Although RYGB that is performed in heavier patients can be more technically difficult, it also yields long-lasting weight loss ${ }^{(3)}$.

On the other hand, LSG is a technically simpler procedure with a shorter post-operative hospitalization and fewer major complications, even in the case of super obese patients (BMI $>60$ $\mathrm{kg} / \mathrm{m} 2)^{(3)}$.

LSG may require a second intervention if the initial weight loss is unsatisfactory ${ }^{(2)}$. Moreover, recent studies have shown that LSG provides effective weight loss outcomes without the second malabsorptive step that occurs with the duodenal switch $^{(3)}$.

In a similar way, isolated single step RYGB has also shown positive weight loss outcomes in super obese patients. Overall, additional data are needed to examine postsurgical outcomes as well as to formulate recommendations regarding the surgical care of super-super obese patients ${ }^{(3)}$.

\section{AIM OF THE WORK}

This work is aiming to focus light on super obese patient; considering the advantages, the disadvantages and to determine long-term outcome of different plans of management regarding the recent guidelines for this group of patients.

\section{PATIENTS AND METHODS}

Patients:

This prospective study was conducted in Al-Azhar University hospitals, Cairo, Egypt in general surgery department. It included 60 obese patients with type $2 \mathrm{DM}$ and randomly divided using closed envelopment method into two groups:

Group (1): 30 patients treated by laparoscopic Sleeve gastrectomy.

Group (2): 30 patients treated by laparoscopic Mini-Gastric Bypass.

\section{Inclusion criteria}

The patients included in this study fulfilled the following criteria:

1- Their age $>18$ years old.

2- Their body mass index ( BMI) $\geq 30$

3- Type $2 \mathrm{DM}$

\section{Exclusion criteria}

The patients who were excluded from the study:

- Endocrine abnormalities: e.g. hypothyroidism, Cushing syndrome.
- Previous bariatric operations.

- Major upper abdominal surgery.

- Type 1 DM.

- Age below 18 years old.

- Pregnant or lactating females.

- Patient with contraindications for insufflation as those with sever cardiovascular or sever restrictive respiratory diseases.

- Patient with significant abdominal ventral hernia.

- Patient with major psychiatric illness.

\section{Methods}

\section{Preoperative workup:}

All patients were subjected to the following:

\section{Complete history taking:}

1- Personal history: as age, sex, marital status.

2- Feeding history and if the patients likes sweet much or not.

3- Duration of obesity.

4- History of previous trials of weight loss whether surgical or non-surgical.

5- Medical history for comorbidities:

- DM: type, onset, course, duration, current medications, controlled or not, if change from oral hypoglycemic to insulin and when, family history.

- Hypertension.

- Cardiac and respiratory problems.

- Family history of obesity.

- Previous deep venous thrombosis (DVT).

- Any other morbidities.

6- Past surgical history.

7- Complete physical examination:

1- Measurement of weight per $\mathrm{Kg}$, height per meter then calculation of $\mathrm{BMI}=$ (weight $\mathrm{Kg} /$ height $\mathrm{m}^{2}$ ).

2- Type of obesity (android or peripheral).

3- Abdominal examination for (scar for pervious surgery, hernia orifices, organomegaly, right hypochondrial tenderness).

4- Cardiac and pulmonary evaluation.

5- Medical consultation for proper control of blood sugar (pre and postoperative).

\section{Investigations:}

Laboratory investigation:

Complete blood count, Liver function test, kidney function test, fasting blood sugar, 2hours Postprandial blood sugar, serum insulin level, Cpeptide level, HbA1c, coagulation profile, serum Calcium, $\mathrm{Na}, \mathrm{K}, \mathrm{Mg}$, , thyroid function tests, serum cortisol level.

\section{Other investigation:}


Chest X-ray, Abdominal U.S, Pulmonary function test, Echocardiography, and Upper gastrointestinal (UGI) endoscopy (if needed).

Data management and statistical analysis

The collected data was analyzed using Statistical package for Social Science (SPSS) 20.
Mean \pm standard deviation was computed for continuous variables e.g. age. Categorical data was expressed as number and percentage. T-test was used to compare means and Chi-squared test for categorical data. P-value of 0.05 or less was considered statistically significant.

\section{RESULTS}

Table (1): Comparison between group 1 (sleeve gastrectomy) and group 2 (minigastric bypass) as regard personal characteristics

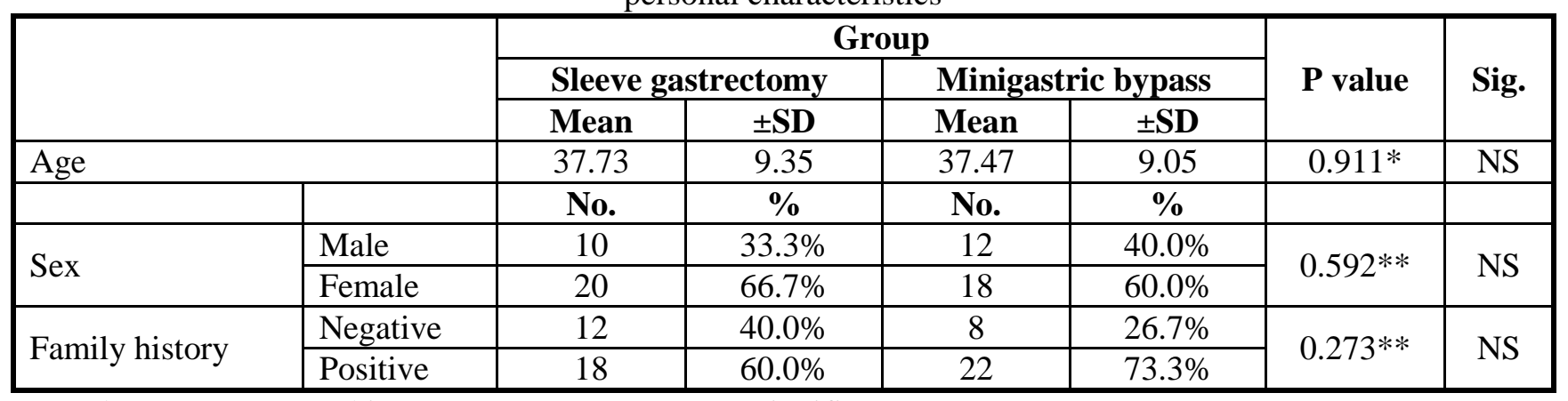

*Student $\mathrm{t}$ test. $\quad * *$ Chi-Square Test. $\quad \mathrm{NS}=$ non significant

Table (2): Comparison between group 1 (sleeve gastrectomy) and group 2 (minigastric bypass) as regard biochemical characteristics

\begin{tabular}{|l|c|c|c|c|c|c|}
\hline \multirow{2}{*}{} & \multicolumn{4}{|c|}{ Group } & \multirow{2}{*}{ P value } & \multirow{2}{*}{ Sig. } \\
\cline { 2 - 5 } & \multicolumn{2}{|c|}{ Sleeve gastrectomy } & \multicolumn{2}{c|}{ Minigastric bypass } & \\
\cline { 2 - 5 } & Mean & $\mathbf{\pm S D}$ & Mean & $\mathbf{\pm S D}$ & & \\
\hline C-peptide & 3.77 & 1.25 & 4.13 & 1.37 & $0.549^{*}$ & NS \\
\hline BMI baseline & 51.93 & 9.78 & 52.33 & 9.41 & $0.421^{*}$ & NS \\
\hline FBS baseline & 145.27 & 12.78 & 149.67 & 12.27 & $0.514^{*}$ & NS \\
\hline HbA1c baseline & 8.21 & 0.88 & 8.10 & 0.92 & $0.326^{*}$ & NS \\
\hline
\end{tabular}

*Student $\mathrm{t}$ test

$\mathrm{NS}=$ non-significant

Table (3): Comparison between group 1 (sleeve gastrectomy) and group 2 (minigastric bypass) as regard medical characteristics

\begin{tabular}{|c|c|c|c|c|c|c|c|}
\hline & \multicolumn{4}{|c|}{ Group } & \multirow{3}{*}{$P$ value } & \multirow{3}{*}{ Sig. } \\
\hline & & \multicolumn{2}{|c|}{ Sleeve gastrectomy } & \multicolumn{2}{|c|}{$\begin{array}{c}\text { Minigastric } \\
\text { Bypass }\end{array}$} & & \\
\hline & & No. & $\%$ & No. & $\%$ & & \\
\hline \multirow{3}{*}{ Distribution of obesity } & Peripheral & 4 & $13.3 \%$ & 8 & $26.7 \%$ & \multirow{3}{*}{$0.235^{*}$} & \multirow{3}{*}{ NS } \\
\hline & Central & 10 & $33.3 \%$ & 12 & $40.0 \%$ & & \\
\hline & Both & 16 & $53.3 \%$ & 10 & $33.3 \%$ & & \\
\hline \multirow{2}{*}{$\begin{array}{l}\text { Duration of } \\
\text { DM (yrs.) }\end{array}$} & $<5$ years & 18 & $60 \%$ & 16 & $53.3 \%$ & \multirow{2}{*}{$0.593^{*}$} & \multirow{2}{*}{ NS } \\
\hline & $>5$ years & 12 & $40 \%$ & 14 & $46.7 \%$ & & \\
\hline \multirow{2}{*}{ Preoperative medication } & $\mathrm{OHG}$ & 22 & $73.3 \%$ & 20 & $66.7 \%$ & \multirow{2}{*}{$0.573^{*}$} & \multirow{2}{*}{ NS } \\
\hline & Insulin & 8 & $26.7 \%$ & 10 & $33.3 \%$ & & \\
\hline \multirow{2}{*}{$\begin{array}{l}\text { Status of DM (according to } \\
\text { baseline HbA1c ) }\end{array}$} & Less Control > 8.5\% & 10 & $33.3 \%$ & 10 & $33.3 \%$ & \multirow{2}{*}{$1.0 *$} & \multirow{2}{*}{ NS } \\
\hline & Better Control $<8.5 \%$ & 20 & $66.7 \%$ & 20 & $66.7 \%$ & & \\
\hline \multirow{2}{*}{ C-peptide } & $<3 \mathrm{ng} / \mathrm{ml}$ & 6 & $20.0 \%$ & 8 & $26.7 \%$ & \multirow{2}{*}{$0.542 *$} & \multirow{2}{*}{ NS } \\
\hline & $>3 \mathrm{ng} / \mathrm{ml}$ & 24 & $80.0 \%$ & 22 & $73.3 \%$ & & \\
\hline
\end{tabular}

*Chi-Square Test $\mathrm{NS}=$ non significant 
Table (4): Comparison between group 1 (sleeve gastrectomy) and group 2 (minigastric bypass) as regard operative time.

\begin{tabular}{|l|c|c|c|c|c|c|}
\hline \multirow{2}{*}{} & \multicolumn{4}{|c|}{ Group } & \multirow{2}{*}{ P value } & \multirow{2}{*}{ Sig. } \\
\cline { 2 - 5 } & \multicolumn{2}{|c|}{ Sleeve gastrectomy } & Minigastric bypass & & \\
\cline { 2 - 5 } & Range & Mean & Range & Mean & & \\
\hline Operative time & $50-120$ min. & 85 & $90-160$ min. & 130 & $0.61^{*}$ & NS \\
\hline
\end{tabular}

*Student $t$ tests. $\quad \mathrm{NS}=$ non significant

Table (5): Comparison between group 1 and 2 as regard FBS at baseline, at follow up and overall FBS change

\begin{tabular}{|l|c|c|c|c|c|c|}
\hline \multirow{2}{*}{} & \multicolumn{4}{|c|}{ Group } & \multirow{2}{*}{ P value } & \multirow{2}{*}{ Sig. } \\
\cline { 2 - 5 } & \multicolumn{2}{|c|}{ Sleeve gastrectomy } & \multicolumn{2}{c|}{ Minigastric bypass } & & \\
\cline { 2 - 5 } & Mean & $\mathbf{\pm S D}$ & Mean & $\mathbf{\pm S D}$ & & \\
\hline FBS baseline & 145.27 & 12.78 & 149.67 & 12.27 & $0.179^{*}$ & NS \\
\hline FBS 3 months & 132.47 & 11.31 & 135.47 & 9.99 & 0.281 & NS \\
\hline FBS 6 months & 124.20 & 10.99 & 125.07 & 10.66 & 0.758 & NS \\
\hline Total FBS change & 21.07 & 11.79 & 24.60 & 5.61 & 0.004 & HS \\
\hline
\end{tabular}

*Student $t$ tests. $\quad \mathrm{NS}=$ non significant

\section{DISCUSSION}

The prevalence of obesity and type 2 diabetes mellitus (T2DM) has increased dramatically worldwide, becoming a serious global health problem. Bariatric surgery is currently the most efficient method for treating obesity, and significant improvement in glycemic control has been observed in individuals with diabetes subjected to this surgery ${ }^{(4)}$.

Given the role of obesity in the etiology of Type 2 diabetes, recent guidelines on diabetes treatment provide that weight loss should be the most logical and cost-effective means of controlling Type 2 diabetes ${ }^{(5)}$.

In addition to weight loss, there are other independent metabolic benefits associated with effects of incretins and possibly other hormonal or neural changes after some surgical procedures which can be integrated in postoperative diabetes control mechanism. For example, rapid and sustained improvements in glycemic control can be achieved within days of gastric bypass surgery, before any significant weight loss is evident ${ }^{(5)}$.

In short-term randomized trials that done by Schauer et al. ${ }^{(6)}$ on 150 obese patients with uncontrolled type 2 diabetes to receive either intensive medical therapy alone or intensive medical therapy plus Roux-en-Y gastric bypass or sleeve gastrectomy, bariatric surgery has been associated with improvement in type 2 diabetes mellitus. The primary end point was a glycated hemoglobin level of $6.0 \%$ or less. At 3 years, the criterion for the primary end point was met by $5 \%$ of the patients in the medical-therapy group, as compared with $38 \%$ of those in the gastric-bypass group ( $\mathrm{P}<0.001)$ and $24 \%$ of those in the sleevegastrectomy group $(\mathrm{P}=0.01)$. The use of glucoselowering medications, including insulin, was lower in the surgical groups than in the medical-therapy group. There were no major late surgical complications.

Sleeve gastrectomy is the first element of the duodenal switch procedure, a variant of the BPD. Lately it became popular as a single procedure because of ease of surgery, relative effectiveness and perceived lack of need for close follow up. A systematic review of the 36 studies available to mid-2009 showed 55\% EWL at 3 years after sleeve gastrectomy ${ }^{(7)}$.

The mini-gastric bypass (MGB) was introduced by Rutledge in 1997 and reported some years later. Since then, thousands of patients have been treated with this approach by several authors in different countries ${ }^{(8)}$.

To date, only Lee et al. ${ }^{(8)}$ have reported a follow-up assessment for MGB patients at 10 years. In their study, MGB proved to be effective in the long term, significantly outperforming even RYGB in several parameters including BMI reduction, resolution of metabolic syndrome, and the need for revision surgery due to both bowel obstruction and internal hernia.

Frequently, MGB is reported as an easier technique, to be preferred to other bariatric approaches, including both RYGB and LSG, for the results in both the short and long terms ${ }^{(8)}$.

The aim of our study was to compare between MGB and LSG in control of type 2 D.M in obese patients through serial postoperative follow up of HBA1c and FBS. The study included 
60 obese patients, 30 of them underwent LSG and the other 30 patients underwent MGB.

The mean operative time of group 1 (sleeve gastrectomy) was 85 minutes ranging between 50-120 minutes and all operations were performed laparoscopically.

The mean operative time of group 2 (minigastric bypass) was 130 minutes ranging between 90 -160 minutes and all operations were performed laparoscopically except one case which was converted to open due to increased airway pressure with abdominal insufflation. The difference between operative time in both groups was statistically non significant.

Compared to the published results, in the study of Musella and his colleagues (to compare between gastric balloon, LAGB, LSG and LMGB) the mean operative time with LSG was $75 \pm 15.3$ minutes while with LMGB mean operative time was $115 \pm 15.6$ minutes ${ }^{(8)}$.

In the study done by Lee and his colleague (which compare between LMGB and LRYGB), the mean operative time with LMGB was 115.3 minutes ${ }^{(9)}$. While in the study done by Gentileschi and his colleague, the mean operative time with LSGB was 58.5 minutes ${ }^{(\mathbf{1 0})}$.

\section{Analysis of postoperative data:}

In LLSG group, it ranged between 1 to 3 days with the mean 2 days while in MGB group, hospital stay ranged between 1 to 5 days with the mean 3 days.

In the study done by Gentileschi et al. ${ }^{(10)}$, the mean postoperative hospital stay after LSG was 3.2 days. While in another study done by Dapri et al. ${ }^{(11)}$, the mean postoperative hospital stay was 3.6 days.

In the study done by Lee and his colleagues, the mean postoperative hospital stay after MGB was 3.7 days ${ }^{(12)}$. While in another study done by Musella et al. ${ }^{(8)}$, the mean postoperative hospital stay was 4 days.

Intraoperative bleeding was nonsignificant in both groups with average $50 \mathrm{cc}$. No case in group 1 (LSG) was converted to open while one case in group 2 (MGB) had been converted to open. Wound infection was reported in one case in MGB group but not reported in LSG group. Chest infection was reported in two cases in LSG group and four cases in MGB group. Two cases in LSG group complained of gastritis induced vomiting while in MGB group, two cases developed biliary gastritis manifestation and resolved conservatively. As regard gall stone disease, one case in each group developed postoperative gall stone and planned for cholecystectomy. Leakage rate and mortality rate was $0 \%$.

The mean BMI loss after one year in MGB $\left(19.67 \pm 7.17 \mathrm{~kg} / \mathrm{m}_{2}\right)$ was more than BMI loss in LSG $(18.47 \pm 5.14 \mathrm{~kg} / \mathrm{m} 2)$ but this difference was statistically non-significant. In comparing to the study of Milone et al. ${ }^{(13)}$ (to compare between LSG and MGB in diabetes remission after one year) in which, LSG and MGB were associated with changes in BMI $(20.33 \pm 4.48 \%$ vs $19.19 \pm$ $4.42 \%)$ and also, the difference between them was statistically non-significant $(\mathrm{P}$ value $=0.931)$.

MGB has a better effect than LSG in diabetes remission detected by that the mean FBS drop after one year in MGB $(37.80 \pm 6.41 \mathrm{mg} / \mathrm{dl})$ was more than after LSG $(29.93 \pm 12.84 \mathrm{mg} / \mathrm{dl})$ and this difference of drop was highly statistically significant ( $\mathrm{p}$ value $<0.004$ ).

At the 12 months follow-up, $66.7 \%$ of subjects who underwent LSG achieved diabetes remission vs $87.5 \%$ of those who underwent MGB $(\mathrm{P}=0.220)$. High preoperative HBA1c was determined to be a negative predictor of diabetes remission at 12 months while there was significant correlation between percent of BMI loss and diabetes remission in both operations ${ }^{(13)}$. The result of our study agreed with this study as regard MGB has better and faster effect on diabetes remission than LSG although the effect of both operation at 6 months are better in Milone study than our study.

The study also revealed positive correlation between BMI loss and diabetes remission but statistically non significant (may indicating presence of other more important mechanisms of postoperative diabetes resolution rather than weight loss).

Also, in the study of Milone et al. ${ }^{(13)}$, high preoperative HBAlc was determined to be a negative predictor of diabetes remission at 12 months in both operation LSG and MGB. Also, significant correlations were not detected in the percent change from baseline to 12 months follow up between BMI and blood glucose level in both operation $(\mathrm{P}=0.673$ for $\mathrm{LSG}, \mathrm{P}=0.071$ for MGB), as well as between BMI and $\mathrm{HbA1c}$ changes $(\mathrm{P}=0.202$ for $\mathrm{LSG}$ and $\mathrm{P}=0.946$ for MGB). Also, our study didn't find statistically significant correlation between BMI loss and diabetes resolution in both operations.

Ghrelin hormone has an anti-insulin effect so postoperative reduction in ghrelin hormone (by 
excision of fundus in LSG, exclusion of it in MGB) improves insulin sensitivity ${ }^{(\mathbf{1 4})}$.

After gastric bypass, bypassing and exclusion of proximal bowel (duodenum and proximal jejunum which are the main site of antincretin hormones) from nutrient, leads to reduction in levels of ant-incretin hormones and so, improve insulin secretion and glycemic control. The superiority of MGB on LSG in diabetes remission may be mainly attributed to the foregut mechanism (beside the more prominent hindgut theory of MGB).

Finally our study suggests that bariatric surgery (mainly LSG and MGB which are the most common bariatric operations nowadays) is considered the best long term treatment of diabetes type 2 in obese patients and MGB is superior to LSG in diabetes remission.

\section{CONCLUSION}

So in conclusion, bariatric surgery (LSG and MGB) is not only weight reducing surgery but a metabolic surgery which can cure most of the metabolic syndrome and they are considered the most effective long term treatment modality for type 2 diabetes in obese patients and with comparing between LSG and MGB, our study suggests that MGB has better and earlier effect than LSG in diabetes remission.

\section{REFERENCES}

1. Eisenberg D, Bellatorre A, Bellatorre $\mathbf{N}$ (2013): Sleeve gastrectomy as a stand-alone bariatric operation for severe, morbid, and super obesity. JSLS: Journal of the Society of Laparoendoscopic Surgeons, 17(1):63.

2. Zerrweck C, Sepúlveda EM, Maydón HG, Campos F, Spaventa AG, Pratti V, Fernández I (2014): Laparoscopic gastric bypass vs. sleeve gastrectomy in the super obese patient: early outcomes of an observational study. Obesity surgery, 24(5):712-7.

3. Lemanu DP, Srinivasa S, Singh PP et al. (2012): Optimizing perioperative care in bariatric surgery patients. Obesity surgery, 22(6):979-90.
4. Reis CE, Alvarez-Leite JI, Bressan J, Alfenas RC (2012): Role of bariatric-metabolic surgery in the treatment of obese type 2 diabetes with body mass index $<35 \mathrm{~kg} / \mathrm{m} 2$ : a literature review. Diabetes technology \& therapeutics, 14(4), 365-372.

5. Dixon JB, Zimmet P, Alberti KG et al. (2011): Bariatric surgery: an IDF statement for obese Type 2 diabetes. Diabetic Medicine, 28(6):628-42.

6. Schauer PR, Bhatt DL, Kirwan JP et al. (2014): Bariatric Surgery versus Intensive Medical Therapy for Diabetes - 3-Year Outcomes. N Engl J Med., 370(21): 2002-13.

7. O'Brien PE (2010): Bariatric surgery: Mechanisms, indications and outcomes. Journal of Gastroenterology and Hepatology, 25: 1358-1365.

8. Musella M, Susa A, Greco F et al. (2014): The laparoscopic mini-gastric bypass: the Italian experience: outcomes from 974 consecutive cases in a multicenter review Surg Endosc., 28:156-163.

9. Lee WJ, Ser KH, Lee YC et al. (2012): Laparoscopic Roux-en-Y vs. mini-gastric bypass for the treatment of morbid obesity: a 10-year experience. Obes Surg., 22:1827-1834.

10. Gentileschi P, Camperchioli I and D'Ugo S et al. (2012): Staple line reinforcement during laparoscopic sleeve gastrectomy using three different techniques: randomized trial. Surg Endosc., 26: 2623-9.

11. Dapri G, Cadiere GB, Himpens J (2010): Reinforcing the staple line during laparoscopic sleeve gastrectomy: prospective randomized clinical study comparing three different techniques. Obes Surg., 20:462-7.

12. Lee CW, Kelly JJ, Wassef WY et al. (2005): Complication of bariatric surgery. Curr Gastroenterol., 23(6): 636-643

13. Milone M, Minno N, Leongito $M$ et al. (2013): Bariatric surgery and diabetes remission: Sleeve gastrectomy or mini-gastric bypass? World J Gastroenterol., 19(39): 6590-6597.

14. Barazzoni $\mathrm{R}$, Zanetti $M$, Ferreira $\mathrm{C}$ et al. (2007): Relationships between desacylated and acylated ghrelin and insulin sensitivity in the metabolic syndrome. Journal of Clinical Endocrinology \& Metabolism, 92: 3935-3940. 